\title{
ARTICLE
}

\section{Image guided tumour ablation}

\author{
A R Gillams \\ Department of Medical Imaging, The Middlesex Hospital, Mortimer Street, London, W1T 3AA, UK \\ Corresponding address: Dr A Gillams, Department of Medical Imaging, The Middlesex Hospital, Mortimer Street, \\ London, WIT 3AA, UK. E-mail: a.gillams@medphys.ucl.ac.uk
}

Date accepted for publication 5 April 2005

\begin{abstract}
Several different technologies have been employed for the local ablation of tissue by thermal techniques. At the present time the most widely favoured technique is radiofrequency ablation (RFA) but developments in other techniques, e.g. microwave may change this. In many countries RFA or percutaneous ethanol injection (PEI) are accepted therapies for patients with Childs Pugh Class A or B cirrhosis and early hepatocellular carcinoma (HCC). Results for RFA in large series of patients with liver metastases from colon cancer are very promising. Five-year survival rates of $26 \%$ from the time of first ablation and $30 \%$ from the diagnosis of liver metastases for patients with limited $(<6,<5 \mathrm{~cm})$ liver disease who are not surgical candidates compares well with post resection series where 5-year survival rates vary between $29 \%$ and $39 \%$ in operable candidates. Sufficient experience has now been gained in lung and renal ablation to show that these are minimally invasive techniques which can produce effective tumour destruction with a limited morbidity. More novel areas for ablation such as adrenal or pelvic recurrence are being explored.
\end{abstract}

Keywords: Radiofrequency ablation; interstitial laser therapy; liver tumours.

\section{Introduction}

Image guided tumour ablation is one of the most exciting but also one of the more challenging new developments in radiology. Tumour ablation requires a shift in emphasis towards clinical care yet good interventional and diagnostic skills are essential as well. The first description of percutaneous ethanol injection (PEI) was published in 1986, a small series of just 14 patients with small, non-resectable hepatocellular carcinoma $^{[1]}$. Thermal techniques were first performed in the liver at our institution in 1989, initially using single bare tip neodymium yttrium aluminium garnet (NdYAG $\lambda 1064 \mathrm{~nm}$ ) laser fibres placed under US guidance ${ }^{[2]}$. These early reports of ablation efficacy are now being confirmed with large series and clinically relevant followup. Randomised controlled trials are ongoing. The role of radiofrequency in hepatocellular carcinoma (HCC) is the best established but both renal and lung applications are developing fast and even the most conservative liver resection surgeon is starting to accept that there are patients with metastatic disease who will benefit from local ablation. This paper looks at some of the technical details, followed by a discussion of the major applications and then a summary of the latest literature in the most rapidly developing areas.

\section{Technical aspects}

Energy sources can be grouped into four major categories: those employing thermal energy, either heating or cooling; direct injection therapies; photodynamic therapy; and ionizing radiation. In this article we concentrate on the thermal techniques and percutaneous ethanol ablation.

\section{Thermal energy}

With all the heating techniques the aim is to raise the temperature of the tissue to be destroyed to between 60 and $100^{\circ} \mathrm{C}$. This is to produce coagulative necrosis

This paper is available online at http://www.cancerimaging.org. In the event of a change in the URL address, please use the DOI provided to locate the paper. 
yet avoid charring and vaporization of tissue. There are five thermal techniques: radiofrequency (RF), laser, microwave, cryotherapy and high intensity focused ultrasound $^{[3]}$.

\section{Radiofrequency}

RF current induces ionic agitation that in turn results in heating. RF has been used for many years to perform electrocautery in the operating room or to produce discrete, focal lesions that interrupt aberrant cardiac conduction pathways. Initial electrodes were unipolar of low power, $<50 \mathrm{~W}$, and were not internally cooled. RF technology now uses arrays of electrodes that are activated simultaneously, internally watercooled electrodes, high power generators $<250 \mathrm{~W}$ and simultaneous perfusion of the tissue with saline ${ }^{[4-9]}$. Currently there is a lot of interest in bipolar electrodes, which historically produced only small discrete lesions but have now been successfully modified for use in the liver ${ }^{[10]}$. Energy deposition and therefore heating should be more predictable with bipolar systems.

\section{Laser}

Both neodymium yttrium aluminium garnet (NdYAG $\lambda$ $1064 \mathrm{~nm})$ and solid state lasers $(\lambda 805 \mathrm{~nm})$ have been used successfully in tumour ablation. Photon absorption and heat conduction produce hyperthermia and coagulative necrosis. Laser energy is delivered through flexible thin fibres of diameter 400-600 $\mu \mathrm{m}$. Fibre morphology can be varied depending on the area to be treated. A point source from a bare tip fibre will produce a sphere of necrosis, whereas a diffuser fibre will produce an elliptical ablation. As with radiofrequency both water cooling and increased power, up to $40 \mathrm{~W}$ have been introduced to good effect. Although more expensive to set up and support than RF, laser ablations are a little more predictable.

\section{Microwave}

Microwaves (2450 MHz) cause rotation and vibration of water molecules thus producing heat. Initial electrodes were too large for percutaneous use and required laparotomy, this is no longer the case. The equipment consists of a generator and a monopolar needle electrode which is introduced through a $14 \mathrm{G}$ access needle. Multiple percutaneous electrodes are generally required. Each microwave application produces a discrete focus of necrosis, e.g. a single treatment for $120 \mathrm{~s}$ at $60 \mathrm{~W}$ provides approximately $1.6 \mathrm{~cm}$ of necrosis. For this reason microwave ablation has most often been used for the treatment of small $(<3 \mathrm{~cm})$ hepatocellular carcinoma (HCC). Again water-cooled microwave devices are under development and should improve the amount of ablation that can be achieved.
Cryotherapy

Cryotherapy uses repetitive freezing and thawing of tissue to produce necrosis. Irreversible tissue destruction occurs at temperatures below -20 to $30^{\circ} \mathrm{C}$. Liquid nitrogen and argon gas are used as coolants. Traditional cryotherapy probes have been large requiring laparotomy access for the treatment of liver tumours. Recent advances have seen the development of percutaneous cryotherapy probes of less than $2.5 \mathrm{~mm}$ in diameter. Cryoprobes of different sizes and shape are available to map the morphology of the area to be treated. The development of the ice ball can be monitored using US or MR guidance with an accuracy of 1-5 mm. Most cryotherapy has been applied to tumours of the liver and prostate.

\section{High intensity focused ultrasound}

High intensity focused ultrasound (HIFU) uses frequencies of $0.8-3.2 \mathrm{MHz}$ and focal peak intensities of $5000-20000 \mathrm{~W} / \mathrm{cm}^{2}[11]$. The basic mechanism is heat induced coagulative necrosis. Pathological studies demonstrate specific damage to vessels including tumour micro-vasculature ${ }^{[12]}$. HIFU has the advantage of being a trackless technique, performed without anaesthesia and with no risk of tumour seeding. A clear acoustic path from skin to the tissue to be ablated is required. The technique has been available since the 1940s but improvements in imaging and in HIFU technology, e.g. variable focusing and electronic beam steering, have renewed interest in the technique. The main problem is the limited amount of necrosis that can be achieved per unit time; treatment times can run to several hours making general anaesthesia mandatory. In the liver; techniques for improving the efficacy of HIFU include resection of overlying ribs and collapse of the right lung to reduce liver movement during treatment.

\section{Direct injection therapies}

Ethanol, acetic acid and gel stabilized chemotherapeutic agents have all been used to ablate tumours with variable success. Gene therapy is hypothesised as the therapy of tomorrow.

\section{Percutaneous ethanol injection}

Percutaneous ethanol injection (PEI) was one of the first effective ablative techniques to be widely adopted for the treatment of small HCCs. Ethanol causes dehydration and subsequently necrosis ${ }^{[13]}$. Under US guidance a fine needle $(21-22 \mathrm{G})$ is introduced into the tumour and 95\%-100\% ethanol injected. To achieve complete ablation the ethanol must reach all parts of the tumour, however, ethanol spreads unevenly and the needle needs 
to be repositioned accordingly. Ethanol can reflux along the needle tract and cause pain; this limits the amount that can be injected at any one time in the conscious patient. PEI is therefore either performed as a multistage, outpatient technique under conscious sedation or as a single stage procedure under general anaesthesia. PEI is most effective in encapsulated HCC and of little benefit in infiltrating $\mathrm{HCC}$ or in metastases. Thermal techniques are preferred for the treatment of metastases. In HCC, thermal techniques provide more necrosis in less time and in fewer sessions. PEI still has a role in the treatment of HCC not amenable to RF, e.g. exophytic lesions which can rupture, with disastrous consequences, during heating. Some centres prefer PEI for small lesions $(<2 \mathrm{~cm})$ because of the relative ease of implementation and lower cost.

\section{Applications to specific organs}

\section{Liver tumours}

More clinical experience has been gained in the treatment of liver tumours than in any other area. All the thermal techniques have been tried in the liver. Today, PEI has been replaced by laser or RF ablation in most centres. Although both of these latter techniques are effective, currently RF is the preferred technique and the one most widely practiced. Laser has the advantage of being more MR compatible permitting direct MR monitoring ${ }^{[14]}$. MR compatible RF electrodes are now available and there are recent reports of successful simultaneous ablation and MR monitoring which has previously been a stumbling block. Comparisons of laser and RF suggest that larger volumes of ablation and in particular the ablation of a margin of normal liver has been easier and quicker to achieve with $\mathrm{RF}^{[15]}$. Cryoablation whilst effective carries a higher complication rate. Comparisons of laparoscopic RF with cryoablation have shown a lower complication rate with $\mathrm{RF}^{[16]}$. One study reported a $40.7 \%$ complication rate with cryotherapy as compared to $3.3 \%$ with $\mathrm{RF}^{[17]}$. Comparisons of percutaneous cryotherapy and RF have shown a higher complete ablation rate with $\mathrm{RF}^{[18]}$. Microwave has been used particularly in China and a few centres have performed PDT. HIFU suffers from respiratory motion, long treatment times, limited depth of penetration and reflection by overlying ribs or lung. Therefore, RF is currently the preferred technique for liver tumour ablation.

\section{Pathological validation of $R F$}

Efficacy of RF has been validated in at least two cohorts of patients who subsequently underwent surgical resection ${ }^{[19,20]}$. Pathological specimens that were resected immediately after ablation showed irreversible cell damage with absence of enzymatic activity. RFA induced necrosis fundamentally differs from ordinary tissue death. When cells are lethally injured membrane dysfunction and release of enzymes degrades the cellular components. The entire process takes hours to days followed by weeks of tissue repairing. In heat ablation the affected structures are subjected to instantaneous thermal fixation, equivalent to that produced by formalin. Thermally induced deactivation of the enzyme protein prevents the cell degradation seen in traditional coagulation necrosis. Consequently, with conventional staining techniques, the tissue architecture and cytologic details appear well preserved, despite absence of any activity on enzymatic histochemical assays. However, the loss of enzymatic activity does not exclude antigenicity of the denatured enzyme proteins which explains positive results in RFA lesions on immunohistochemistry. The pronounced vascular destruction associated with RFA often postpones healing by blocking neutrophils with hydrolytic enzymes from access into the lesion. This can result in incomplete absorption and fibrous encapsulation of the residual lesion. Lack of cell viability can be determined early after RFA by a negative reaction with enzymatic histochemical stains, e.g. lactate-dehydrogenase or maleate-dehydrogenase, and nicotinamide adenine dinucleotide-diaphorase ${ }^{[21]}$.

\section{Colorectal metastases}

Limited colorectal liver metastases are the most commonly treated metastatic lesion. The liver is often the first and only site for metastases. There is good evidence that most patients will succumb from their liver metastases and therefore local control can improve life expectancy. This has been the reasoning behind hepatic resection. Surgical resection is the accepted first line treatment for patients with resectable disease. Five-year survival figures post resection range from $25 \%$ to $39 \%{ }^{[22]}$. Traditionally most patients $(80 \%-90 \%)$ are not candidates for surgical resection due to the extent or distribution of disease or concurrent medical disability ${ }^{[23]}$. Although historical chemotherapy results have been disappointing, recent results are better. Irinotecan has resulted in the first significant improvement in survival to a median 17.4 months and 1-year survival of $69 \%{ }^{[24]}$. Improved response rates have been achieved with Oxaliplatin, 53\% as compared to $28 \%$ with $5 \mathrm{FU}$ and folinic acid ${ }^{[25]}$. The improvement in survival can be increased further by successive Irinotecan and Oxaliplatin. There are both surgical and RF papers showing that down staging with neoadjuvant chemotherapy followed by ablation or resection is useful ${ }^{[26,27]}$.

\section{Ablation results}

Between 1993 and 1995 we performed laser thermal ablation and reported a median survival of 27 months ${ }^{[28]}$. Early results using RF instead of bare tip fibre laser 
showed a median survival of 34 months and 3-year survival of $36 \%$ from the time of thermal ablation ${ }^{[29,30]}$. Latterly we have published our results in 167 patients. For patients with $\leq 5$ metastases, maximum diameter $\leq 5 \mathrm{~cm}$ and no extra-hepatic disease, the 5-year survival from the time of diagnosis was $30 \%$ and from the time of first thermal ablation was $26 \%{ }^{[31]}$.

Thirty percent survival at 5 years in inoperable patients compares reasonably well with 5-year survival of $29 \%$ $39 \%$ for patients undergoing resection for operable disease $^{[32,33]}$. Other thermal ablation groups have reported similar survival results ${ }^{[34,35]}$. Our current recommendation is to accept patients with five metastases or less and a maximum diameter of $5 \mathrm{~cm}$ but we are also studying RF ablation in patients with as many as nine tumours with a maximum diameter of no more than $4.5 \mathrm{~cm}$.

Where the distribution of disease is not amenable to surgery, the use of a combination of RF and resection can be considered. For those with concurrent disability, $\mathrm{RF}$ is a much less invasive alternative than surgery with lower complication rates. Other applications for RF include patients with limited liver disease who have insufficient residual liver to allow resection, usually post hemi-hepatectomy patients with new metastases in the residual lobe. RF like surgery is most effective in small tumours. The ideal RF candidate is often the ideal surgical candidate. Retrospective comparisons of $\mathrm{RF}$ and repeat hepatic resection show similar survival benefits ${ }^{[36]}$. At our institution, a retrospective comparison of RF and surgery in solitary metastases showed a similar survival rate ${ }^{[37]}$. However, until we have prospective trials comparing the two treatments, surgery will remain the first line treatment for those who are suitable. RF can be performed either concurrently or sequentially to chemotherapy and can be repeated if new lesions or recurrence occurs. If the patient develops more extensive disease such that RF can no longer be performed then chemotherapy should be considered.

From the preceding discussion it becomes clear that patients with colorectal liver metastases may undergo resection, RF ablation and chemotherapy at different times during the course of their disease. Ideally these patients should be able to move smoothly between the specialties and receive the treatment appropriate to their disease status. Randomized, controlled trials are now in process. The European CLOCC trial aims to compare the effect of ablation in conjunction with systemic chemotherapy with systemic chemotherapy alone in patients with inoperable colorectal metastases.

\section{Hepatocellular carcinoma}

Unlike liver metastases local ablative therapy is well established in HCC. Historically ablation was performed using pure ethanol. Trials of percutaneous ethanol injection (PEI) and liver resection suggest a comparable survival. In one trial, Childs Pugh Class A patients had a 3-year survival of $71 \%$ following PEI compared to $79 \%$ following surgery and Childs Pugh Class B patients had a 3-year survival of $41 \%$ and $40 \%$, respectively ${ }^{[38]}$. RF has been compared to PEI in a randomized trial setting and shown to have a higher complete ablation rate in fewer treatment sessions but also a higher complication rate $^{[39]}$. A prospective randomized controlled trial of RF and PEI in early HCC showed a significant event free and tumour free survival at 2 years with a trend towards an overall improvement in survival ${ }^{[40]}$. Microwave has been used but again comparative studies suggest that RF is more efficacious ${ }^{[41]}$. Encapsulated HCC is generally easier to destroy than metastases as the heat is contained and amplified within the lesion, a phenomenon known as the 'oven effect'. Several centres use laser effectively in the treatment of $\mathrm{HCC}$; to date there has been no comparison of laser and $\mathrm{RF}$ in $\mathrm{HCC}^{[42]}$. Current recommendations for RF in $\mathrm{HCC}$ are Childs Pugh Class $\mathrm{A}$ or $\mathrm{B}$ cirrhosis and no more than three lesions, no larger than $3.0 \mathrm{~cm}$ or one nodule $<5 \mathrm{~cm}^{[43]}$. Screening programmes, for the detection of early $\mathrm{HCC}$ in patients with hepatitis $\mathrm{C}$ or $\mathrm{B}$, are not widespread and therefore many patients present with large tumours. Although transarterial chemoembolisation (TACE) alone has not yet been shown to have a significant impact on survival, the combination of selective TACE and thermal ablation has been explored with some success in this cohort ${ }^{[44,45]}$. Different techniques have been used, e.g. laser followed weeks later by TACE, or balloon occlusion of the hepatic artery during RF followed by selective catheterization of the tumour feeding vessels and chemoembolization immediately afterwards. It is not yet known what the optimal sequence and timing of the two therapies will be.

\section{Complications}

The complication rate following ablation varies from $2 \%$ to $10 \%$ and the mortality rate is less than $0.8 \%{ }^{[46,47]}$. This is very competitive with alternative treatments. Complications following ablation are the subject of a separate article to be published shortly and therefore further details are not given here.

\section{Lung tumours}

Reports of CT guided thermal ablation suggest that small, $<3.0 \mathrm{~cm}$, tumours can be treated with an acceptable complication rate. Both inoperable primary and limited numbers (usually $<3 /$ lung) of metastatic tumours have been treated. CT fluoroscopy, whilst not essential, can facilitate electrode placement as small, scirrhous lung lesions can be difficult to penetrate with a large calibre needle ${ }^{[48]}$. Given the dwell time for treatment (20$30 \mathrm{~min}$ ) and the calibre of RF electrodes (14-17 G) the pneumothorax rate is not that high. In the largest reported series, a questionnaire study from seven different centres, the pneumothorax rate was $30 \%$ with $<10 \%$ 
requiring temporary intercostal drainage ${ }^{[49]}$. In the same series the incidence of pleural effusion requiring drainage was $<10 \%$; there were two deaths. Other potential complications include infection, haemorrhage (which can be severe) and bronchopleural fistulae. CT scans performed during treatment often demonstrate oedema with or without pulmonary contusion. The presence of a ground glass opacification that completely envelops the lesion is said to indicate complete ablation (Fig. 1). Post treatment CT scans will commonly show pleural effusions, reaction or thickening. There is often some associated consolidation and cavitation occurs in as many as $24 \%{ }^{[50]}$. Over time, successfully treated lesions shrink in size to a scar and may disappear. In one study of colorectal metastases 17/40 (43\%) had disappeared on follow-up CT at 1 year ${ }^{[51]}$. In addition to size criteria, nodule densitometry is also useful in the follow-up of ablated lung tumours. Suh et al. reported a fall from $47 \mathrm{HU}$ enhancement at baseline to a mean of $9 \mathrm{HU}$ at $1-2$ months in 19 tumours in 12 patients ${ }^{[52]}$. Successful ablation is size dependent with one study reporting complete ablation on follow-up fluoro-deoxy-glucose PET and CT in $69 \%$ of tumours smaller than $3 \mathrm{~cm}$ in diameter but in only $39 \%$ of tumours $>3 \mathrm{~cm}^{[53]}$.

\section{Tumours of the kidney}

Nephron sparing surgery presents a challenge to the surgeon. Multiple renal tumours are not rare and can be difficult to resect without complication. Renal tumours up to $3 \mathrm{~cm}$ in diameter can be destroyed in situ by laser or radiofrequency techniques with virtually no damage to the surrounding normal renal tissue. Following a number of promising early reports ${ }^{[54-56]}$, intermediate follow-up data have now been published. In the largest series to date RFA was performed in 42 tumours in 34 patients. Success depended on tumour location and size. Complete ablation was achieved in exophytic tumours, located away from the central sinus, with diameters up to $5 \mathrm{~cm}$. Complete ablation was harder to achieve in centrally placed tumours adjacent to the renal sinus but was achieved in 5/11 ${ }^{[57]}$. Another series, which used either laparoscopic or percutaneous RFA, achieved complete ablation, as defined by absent enhancement in the tumour bed at 12 months follow-up, in 23/24 with importantly no change in serum creatinine ${ }^{[58]}$. Initial concerns that adjacent bowel or pleura would prevent a percutaneous approach have been allayed by the use of $5 \%$ dextrose instilled around the tumour to displace adjacent structures that could be injured.

\section{Extra-hepatic, extra-renal, intra-abdominal ablation}

Where nodal or local recurrence has occurred despite maximal radiotherapy treatment, local ablative techniques
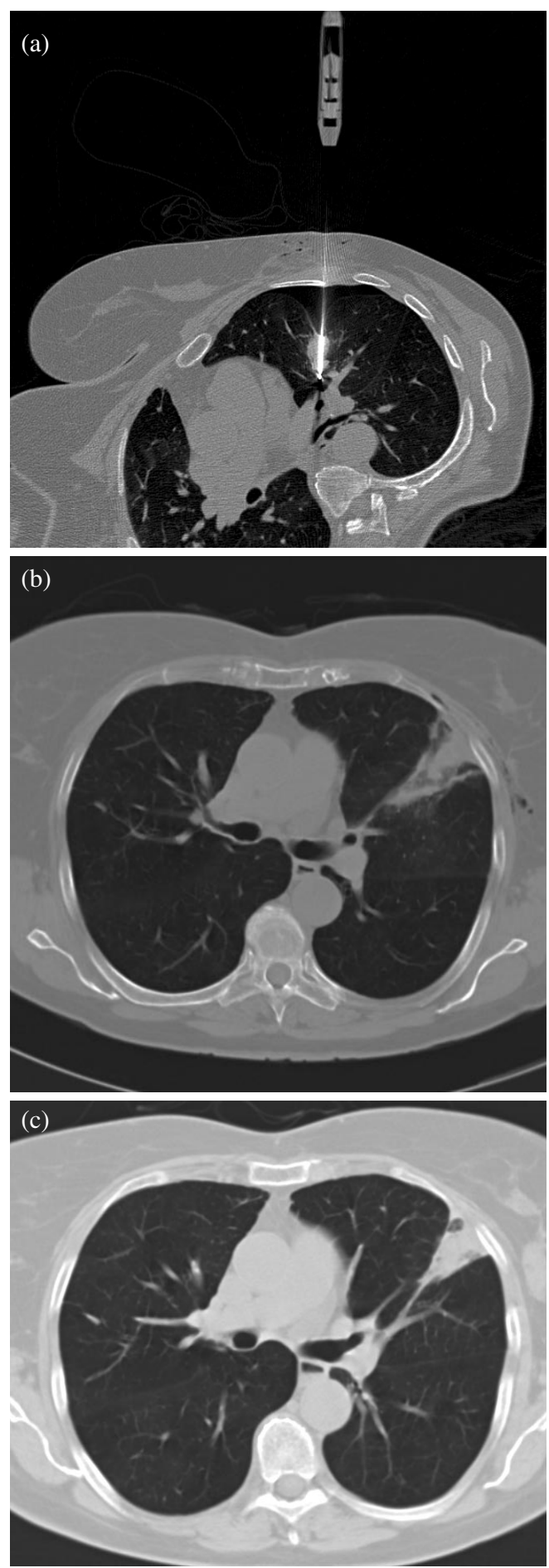

Figure 1 (a) CT scan showing a single, water cooled electrode inserted percutaneously with the tip beyond the lung tumour prior to heating. Note invagination of the pleura. (b) CT scan the following day shows ground glass opacification that embraces the entire tumour-a good indicator of the ablation zone. There is no pneumothorax but there is surgical emphysema in the subcutaneous tissues over the left lateral chest wall. (c) CT scan 2 months later showing a wedge shaped opacification at the treatment site that is already starting to reduce in size. 
can be effective ${ }^{[59]}$. Five percent dextrose may need to be instilled between the treatment zone and any vulnerable structures, e.g. colon, to prevent collateral injury. To avoid neural damage, some centres place a thermosensor adjacent to the structure to be protected. Small series of ablation in adrenal tumours have also been published indicating that this is a feasible technique ${ }^{[60,61]}$. In our own experience, ablation of normal adrenal tissue adjacent to the tumour can produce marked hypertension. We therefore advocate intra-arterial line monitoring of all patients undergoing adrenal ablation and the ready availability of vasodilators.

\section{Bone tumours}

Primary bone tumours are usually treated by chemotherapy, radiotherapy and surgery, however, if aggressive therapy is delivered at an early stage recurrence can be very difficult to treat. Treatment by RF ablation may be curative, but is more likely to form part of a palliative treatment regime. CT or MRI are the usual guidance methods.

RF ablation has been advocated in the symptomatic palliation of bone metastases following radiotherapy ${ }^{[62]}$. Initial results suggest that ablation can produce significant reductions in pain levels and analgesic requirements. Only limited numbers of metastases can be treated. It is important to select patients with a clearly defined and understood dominant site of bone pain. The exact mechanism of action is not understood. The aetiology of bone pain in metastases is complex with a variety of tumoural effects (release of chemical mediators, bone destruction, pressure effects, fractures, stretching of the periosteum) contributing to pain. It is thought to be more important to ablate the tumour/bone interface than to debulk the tumour.

\section{Combination therapy and chemoprevention}

The great promise of ablation, namely its focality, precision and minimal invasiveness can be its downfall. Local ablative techniques need to be used carefully, preferably within the context of multi-disciplinary meeting and consensus. It is important to recognize the need for more extensive treatment in those cancers that arise within field change, e.g. the prostate, to combine ablation with systemic therapies for those cancers that are part of a systemic process and to use chemoprevention after successful local therapy in tumours such as HCC.

\section{Conclusion}

Image guided ablation is established in the liver and rapidly developing a role in the management of inoperable lung and renal cancer. There are a whole range of other possible applications to be explored and many more questions to be answered.

\section{References}

[1] Livraghi T, Festi D, Monti F, Salmi A, Vettori C. US-guided percutaneous alcohol injection of small hepatic and abdominal tumors. Radiology 1986; 161: 309-12.

[2] Steger A, Lees W, Walmsley K, Bown S. Interstitial laser hyperthermia: a new approach to local destruction of tumours. Br Med J 1989; 299: 362-5.

[3] Dodd GD 3rd, Soulen MC, Kane RA et al. Minimally invasive treatment of malignant hepatic tumors: at the threshold of a major breakthrough. Radiographics 2000; 20: 9-27.

[4] Lorentzen T. A cooled needle electrode for RF tissue ablation: thermodynamic aspects of improved performance compared with conventional needle design. Acad Radiol 1996; 3: 556-63.

[5] Goldberg SN, Gazelle GS, Dawson SL, Rittman WJ, Mueller PR, Rosenthal DI. Tissue ablation with RF using multiprobe arrays. Acad Radiol 1995; 2: 670-4.

[6] Miao Y, Ni Y, Yu J, Marchal G. A comparative study on validation of a novel cooled-wet electrode for RF liver ablation. Invest Radiol 2000; 35: 438-44.

[7] Miao Y, Ni Y, Mulier S et al. Ex vivo experiment on RF liver ablation with saline infusion through a screw-tip cannulated electrode. J Surg Res 1997; 71: 19-24.

[8] Livraghi T, Goldberg SN, Monti F et al. Saline enhanced RF tissue ablation in the treatment of liver metastases. Radiology 1997; 202: 205-10.

[9] Goldberg SN, Ahmed M, Gazelle GS et al. Radio-frequency thermal ablation with $\mathrm{NaCl}$ solution injection: effect of electrical conductivity on tissue heating and coagulation-phantom and porcine liver study. Radiology 2001; 219: 157-65.

[10] Burdio F, Guemes A, Burdio JM et al. Bipolar saline-enhanced electrode for radiofrequency ablation: results of experimental study of in vivo porcine liver. Radiology 2003; 229: 447-56.

[11] Ter Haar G. High intensity ultrasound. Semin Laparosc Surg 2001; 8: 77-89.

[12] Wu F, Chen WZ, Bai J et al. Pathological changes in human malignant carcinoma treated with high-intensity focused ultrasound. Ultrasound Med Biol 2001; 27: 1099-106.

[13] Livraghi T. Percutaneous ethanol injection in the treatment of HCC in cirrhosis. Hepatogastroenterology 2001; 48: 20-4.

[14] Vogl TJ, Muller PK, Hammerstingl R et al. Malignant liver tumors treated with MR imaging-guided laser-induced thermotherapy: technique and prospective results. Radiology 1995; 196: 257-65.

[15] Lees WR, Gillams A. Comparison of the effectiveness of cooled tip RF and interstitial laser photocoagulation in liver tumour ablation. Radiology 1999; 213P: 122.

[16] Bilchik AJ, Wood TF, Allegra D et al. Cryosurgical ablation and RF ablation for unresectable hepatic malignant neoplasms. Arch Surg 2000; 135: 657-64.

[17] Pearson AS, Izzo F, Fleming RY et al. Intraoperative RF ablation or cryoablation for hepatic malignancies. Am J Surg 1999; 178: 592-9.

[18] Adam R, Hagopian E, Linhares M et al. A comparison of percutaneous cryosurgery and percutaneous RF for unresectable hepatic malignancies. Arch Surg 2002; 137: 1332-9.

[19] Scudamore CH, Lee SI, Patterson EJ et al. RF ablation followed by resection of malignant liver tumors. Am J Surg 1999; 177: 411-7.

[20] Goldberg SN, Gazelle GS, Compton CC, Mueller PR, Tanabe KK. Treatment of intrahepatic malignancy with RF ablation. Radiologic-pathologic correlation. Cancer 2000; 88: 2452-63.

[21] Morimoto M, Sugimori K, Shirato K et al. Treatment of hepatocellular carcinoma with RF ablation: radiologic-histologic 
correlation during follow-up periods. Hepatology 2002; 35 : 1467-75.

[22] Fong Y, Cohen AM, Fortner JG et al. Liver resection for colorectal metastases. J Clin Oncol 1997; 15: 938-94.

[23] Steele G Jr, Ravikumar TS. Resection of hepatic metastases from colorectal cancer. Ann Surg 1989; 210: 127-38.

[24] Douillard JY. V303 Study Group. Irintoecan and high dose fluorouracil/leucovorin for metastatic colorectal cancer. Oncology 2000; 14: 51-5.

[25] Giacchetti S, Perpoint B, Zidani R et al. Phase III multicenter trial of oxaliplatin added to chronomodulated fluorouracilleucovorin as first line treatment of metastatic colorectal cancer. J Clin Oncol 2000; 18: 136-47.

[26] Bismuth H, Adam R, Levi F et al. Resection of nonresectable liver metastases from colorectal cancer after neoadjuvant chemotherapy. Ann Surg 1996; 224: 509-22.

[27] Shankar A, Leonard P, Renaut AJ et al. Neoadjuvant therapy improves respectability rates for colorectal cancer. Ann R Coll Surg Engl 2001; 83: 85-8.

[28] Gillams A, Lees WR. Survival after percutaneous, image guided thermal ablation of hepatic metastases from colorectal cancer. Dis Colon Rectum 2000; 43: 656-61.

[29] Gillams AR, Lees WR. Image guided ablation of colorectal liver metastases: time for a randomized controlled trial vs. hepatic resection. Radiology 1999; 213P: 212.

[30] Gillams AR. Thermal ablation of liver metastases. Abdominal Imaging 2001; 26: 361-8.

[31] Gillams AR, Lees WR. Radio-frequency ablation of colorectal liver metastases in 167 patients. Eur Radiol 2004; 14: 2261-7.

[32] Jenkins LT, Millikan KW, Bines SD, Staren ED, Doolas A. Hepatic resection for metastatic colorectal cancer. Am Surg 1997; 63: 605-10.

[33] Nordlinger B, Guiguet M, Vaillant JC et al. Surgical resection of colorectal carcinoma metastases to the liver. Cancer 1996; 77 : 1254-62.

[34] Solbiati L, Livraghi T, Goldberg SN et al. Percutaneous RF ablation of hepatic metastases from colorectal cancer: long term results in 117 patients. Radiology 2001; 221: 159-66.

[35] Vogl TJ, Straub R, Eichler K, Sollner O, Mack MG. Colorectal carcinoma metastases in liver: laser-induced interstitial thermotherapy-local tumor control rate and survival data. Radiology 2004; 230: 450-8.

[36] Elias D, de Baere T, Smayra $\mathrm{T}$ et al. Percutaneous RF thermoablation as an alternative to surgery for treatment of liver tumour recurrence after hepatectomy. Br J Surg 2002; 89: 752-6.

[37] Oshowo A, Gillams A, Harrison E, Lees WR, Taylor I. Comparison of resection and radiofrequency ablation for treatment of solitary colorectal liver metastases. Br J Surg 2003; 90: 1240-3.

[38] Livraghi T, Bolondi L, Buscarini L et al. No treatment, resection and ethanol injection in HCC: a retrospective analysis of survival in 391 patients with cirrhosis. Italian Cooperative HCC Study Group. J Hepatol 1995; 22: 522-6.

[39] Livraghi T, Goldberg SN, Lazzaroni S et al. Small HCC: treatment with RF ablation vs. ethanol injection. Radiology 1999; 210: 655-61.

[40] Lencioni RA, Allgaier HP, Cioni D et al. Small hepatocellular carcinoma in cirrhosis: randomized comparison of radiofrequency thermal ablation vs. percutaneous ethanol injection. Radiology 2003; 228: 235-40.

[41] Shibata T, Iimuro Y, Yamamoto Y et al. Small HCC: comparison of radio-frequency ablation and percutaneous microwave coagulation therapy. Radiology 2002; 223: 331-7.

[42] Pacella CM, Bizzarri G, Magnolfi F et al. Laser thermal ablation in the treatment of small HCC; results in 74 patients. Radiology
2001; 221: 712-20.

[43] Livraghi T, Goldberg N, Lazzaroni S et al. HCC: RF ablation of medium and large lesions. Radiology 2000; 214: 761-8.

[44] Pacella CM, Bizzarri G, Magnolfi F, Cecconi P et al. Hepatocellular carcinoma: long term results of combined treatment with laser thermal ablation and transcatheter arterial chemoembolization. Radiology 2001; 219: 669-78.

[45] Lencioni R, Cioni D, Donati F, Bartolozzi C. Combination of interventional therapies in HCC. Hepatogastroenterology 2001; 48: 8-14.

[46] Mulier S, Mulier P, Ni Y et al. Complications of RF coagulation of liver tumours. Br J Surg 2002; 89: 1206-22.

[47] Rhim H, Dodd GD 3rd. RF thermal ablation of liver tumours. J Clin Ultrasound 1999; 27: 221-9.

[48] Dupuy DE, Zagoria RJ, Akerly W, Mayo-Smith WW, Kavanagh PV, Safran H. Percutaneous RF ablation of malignancies in the lung. Am J Roentgenol 2000; 174: 57-60.

[49] Steinke K, Sewell PE, Dupuy D et al. Pulmonary radiofrequency ablation-an international study survey. Anticancer Res 2004; 24: 339-43.

[50] Steinke K, King J, Glenn D, Morris DL. Radiologic appearance and complications of percutaneous computed tomographyguided radio frequency-ablated pulmonary metastases from colorectal carcinoma. J Comput Assist Tomogr 2003; 27: 750-7.

[51] Steinke K, Glenn D, King J et al. Percutaneous imaging-guided radiofrequency ablation in patients with colorectal pulmonary metastases: 1-year follow-up. Ann Surg Oncol 2004; 11: 207-12.

[52] Suh RD, Wallace AB, Sheehan RE, Heinze SB, Goldin JG. Unresectable pulmonary malignancies: CT-guided percutaneous radiofrequency ablation-preliminary results. Radiology 2003; 229: 821-9.

[53] Akeboshi M, Yamakado K, Nakatsuka A et al. Percutaneous radiofrequency ablation of lung neoplasms: initial therapeutic response. J Vasc Interv Radiol 2004; 15: 463-70.

[54] Gervais DA, McGovern FJ, Wood BJ, Goldberg SN, McDougall WS, Mueller PR. RF ablation of renal cell carcinoma: early clinical experience. Radiology 2000; 217 : $665-72$.

[55] Ogan K, Jacomides L, Dolmatch BL et al. Percutaneous RF ablation of renal tumours: technique, limitations and morbidity. Urology 2002; 60: 954-8.

[56] Pavlovich CP, Walther MM, Choyke PL et al. Percutaneous RF ablation of small renal tumours: initial results. J Urol 2002; 167: $10-15$.

[57] Gervais DA, McGovern FJ, Arellano RS, McDougal WS, Mueller PR. Renal cell carcinoma: clinical experience and technical success with radio-frequency ablation of 42 tumors. Radiology 2003; 226: 417-24.

[58] Hwang JJ, Walther MM, Pautler SE et al. Radio frequency ablation of small renal tumors: intermediate results. J Urol 2004; 171: 1814-8

[59] Mack MG, Straub R, Eichler K et al. MR guided laser induced thermotherapy in recurrent extra-hepatic abdominal tumours. Eur Radiol 2001; 11: 2041-6.

[60] Mayo-Smith WW, Dupuy DE. Adrenal neoplasms: CT-guided radiofrequency ablation-preliminary results. Radiology 2004; 231: 225-30.

[61] Wood BJ, Abraham J, Hvizda JL, Alexander HR, Fojo T. Radiofrequency ablation of adrenal tumors and adrenocortical carcinoma metastases. Cancer 2003; 97: 554-60.

[62] Callstrom MR, Charboneau JW, Goetz MP et al. Painful metastases involving bone: feasibility of percutaneous CTand US-guided radio-frequency ablation. Radiology 2002; 224: 87-97. 